\title{
A novel Babesia orientalis 135-kilodalton spherical body protein like: identification of its secretion into cytoplasm of infected erythrocytes
}

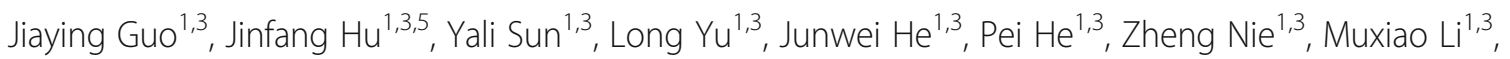

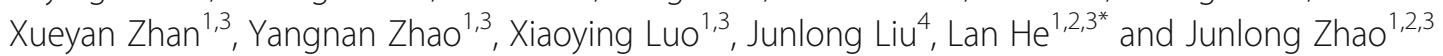

\begin{abstract}
Background: The spherical body is a distinct organelle only existing in Babesia and Theileria. Spherical body proteins (SBPs) are secreted from spherical bodies and incorporated into the cytoplasm of infected erythrocytes during invasion and post-invasion stages. Four different SBP homologues (SBP1, SBP2, SBP3 and SBP4) have been identified in Babesia bovis and Babesia bigemina. So far, there has been no report available about the identification of SBPs in Babesia orientalis.

Methods: The SBP3-like in B. orientalis (BoSBP3-like) was cloned, sequenced, characterized and compared to the SBP3 sequences of B. bovis and B. bigemina by bioinformatics analyses. The BoSBP3-like gene was truncated into three fragments: BoSBP3-like-1 (915 bp), BoSBP3-like-2 (1311 bp) and BoSBP3-like-3 (1011 bp), which were amplified and cloned into the expression vector pET-28a and expressed as three truncated recombinant (His-fusion) proteins. The immunogenicity, native forms and localization of BoSBP3-like were identified by western blot and indirect immunofluorescence assay (IFA).

Results: The BoSBP3-like gene was intronless with an open reading frame (ORF) of 3237 bp, encoded a 1079 amino acid polypeptide with a predicted size of $135 \mathrm{kDa}$, and contained a cysteine-rich region, three dispersing FAINT domains and a signal peptide (1-16 aa) at the N-terminus. The amino acid sequence of BoSBP3-like was 61.6 and $35.0 \%$ identical to that of B. bovis and B. bigemina, respectively. BoSBP3-like was identified as $135 \mathrm{kDa}$ in the parasite lysate by rabbit antiserum against the truncated recombinant BoSBP3-like-1 (rBoSBP3-like-1). Three specific bands corresponding to rBoSBP3-like-1 (1-305 aa, 43 kDa), rBoSBP3-like-2 (306-742 aa, 58 kDa) and rBoSBP3-like-3 (743-1079 aa, $52 \mathrm{kDa}$ ) were detected by reaction with serum from B. orientalis-infected buffalo. The BoSBP3-like was not only localized in the spherical body of $B$. orientalis but also in the cytoplasm of infected erythrocytes of buffalo as puncta-like protein specks at both single and paired parasite development stages.
\end{abstract}

(Continued on next page)

\footnotetext{
* Correspondence: helan@mail.hzau.edu.cn

${ }^{1}$ State Key Laboratory of Agricultural Microbiology, College of Veterinary Medicine, Huazhong Agricultural University, Wuhan 430070, Hubei, China ${ }^{2}$ Key Laboratory of Animal Epidemical Disease and Infectious Zoonoses, Ministry of Agriculture, Huazhong Agricultural University, Wuhan 430070, Hubei, China

Full list of author information is available at the end of the article
} 
(Continued from previous page)

Conclusions: Through secretion into the cytoplasm of infected erythrocytes, BoSBP3-like may play a significant role in adaptation, interaction, and modification related to the host environment to benefit the growth and survival of Babesia. BoSBP3-like could react with the serum from B. orientalis-infected buffalo, but not healthy buffalo, implicating that BoSBP3-like is highly antigenic and may serve as a candidate diagnostic antigen for the detection of $B$. orientalis.

Keywords: Babesia orientalis, Spherical body protein, Erythrocyte, Localization, Cytoplasm

\section{Background}

Babesia spp. are tick-borne haemoprotozoan parasites and taxonomically classified in the phylum Apicomplexa, order Piroplasmida. Babesia spp. can invade a broad range of vertebrate hosts and even humans, causing serious problems in the livestock industry, pet animal health, wildlife protection and human public health throughout tropical and subtropical regions of the world [1, 2]. Babesia orientalis has recently been described based on data concerning parasite's morphology, transmission, pathogenicity and phylogenetic relationships. This parasite is the causative agent of one of the most important diseases of buffalo in central and southern China, characterized by fever, anemia, icterus, hemoglobinuria and even mortality, leading to immense economic losses [3].

The apical complex, the characteristic fine structure for apicomplexan parasites, consists of three specialized secretory organelles, rhoptries, micronemes and dense granules [4] and will release and incorporate proteins into the host environment during invasion and postinvasion $[5,6]$. However, dense granules have not been identified in Babesia and Theileria. There is a unique organelle known as spherical body present in the apical complex of Babesia spp. and Theileria spp. which is a homologue to dense granules [6]. One to four relatively large spherical bodies within each merozoite are located subjacent to the rhoptries and the micronemes [7]. Several spherical body specific proteins (SBP1, SBP2, SBP3 and SBP4) have been only identified and characterized in Babesia bovis and Babesia bigemina [6-10]. All of these spherical body proteins are found localized either in the cytoplasm or cytoplasmic face of the infected red blood cells (iRBC). Similarly, dense granules were characterized to be localized either in the cytoplasmic face of iRBC or within a parasitophorous vacuole (PV). Dense granules were reported to involve in interaction with the host, stabilizing the parasite environment, disrupting infected erythrocytes and triggering host immune responses [11-14]. Despite so many differences between spherical bodies and dense granules, the released proteins may play the same roles during parasite invasion, nutrient intake, waste elimination and adaptation to the host environment.
To date, many efforts have been made to identify SBPs in Babesia and the mechanisms for their interaction with the host, but little information is available in this respect. In this study, we identified and characterized a $135 \mathrm{kDa}$ spherical body protein 3-like of B. orientalis (BoSBP3like). The structure, immunogenicity and localization of BoSBP3-like were investigated by western blot and IFA and compared with those of $B$. bovis and B. bigemina. Integrated results revealed BoSBP3-like as a candidate diagnostic antigen for detection of $B$. orientalis and a vaccine candidate antigen for further related research.

\section{Methods \\ Experimental animals}

Three buffalo (1 year old) were purchased from a Babesia-free area, and were confirmed to be free of Babesia and Theileria by microscopic examination and reverse line blot [15]. Two buffalo were splenectomized two weeks before intrajugular injection of $4 \mathrm{ml}$ of $B$. orientalis (Wuhan Strain)-infected blood with $1 \%$ of parasitized erythrocyte, and the remaining buffalo was used as a control. Blood samples from the two buffalo were collected every day to monitor the parasitaemia until it reached $3 \%$.

Nine Japanese white specific pathogen free (SPF) rabbits $(2.5 \mathrm{~kg}$ each) were used for preparation of immune serum against recombinant BoSBP3-like-1 (rBoSBP3-like-1), recombinant BoSBP3-like-2 (rBoSBP3-like-2) and recombinant BoSBP3-like-3 (rBoSBP3-like-3), and one Japanese white rabbit was used as a control.

\section{Parasite and merozoite antigen}

Babesia orientalis merozoite antigen was prepared as previously described with some modifications [16]. Briefly, the iRBC pellets were washed by phosphate buffer saline (PBS) and then lysed by Tris/EDTA/NaCl (TEN) buffer. The soluble antigen was extracted by centrifugation at $16,300 \times g$ for $30 \mathrm{~min}$ and washed twice in PBS to a final volume of 50-100 $\mathrm{ml}$, then stored at $-20{ }^{\circ} \mathrm{C}$ for further use.

\section{Genomic DNA, RNA extraction and complementary DNA preparation}

The leukocytes of $B$. orientalis-infected blood were removed by using Plasmodipur Filters (EuroProxima, 
Arnhem, the Netherlands). Total RNA was extracted from $400 \mu \mathrm{l}$ of the $B$. orientalis-infected blood using a TRIzol $^{\circ}$ RNA extraction kit (Invitrogen, Carlsbad, CA, USA) according to the manufacturer's instructions. RNA was treated with DNase I (Invitrogen). A complementary DNA (cDNA) was amplified by reverse transcribed PCR (RT-PCR) using a FastQuant ${ }^{\circ}$ RT Kit (Tiangen Biotech, Beijing, China) and stored at $-80{ }^{\circ} \mathrm{C}$ until further analysis.

Genomic DNA (gDNA) was extracted from $200 \mu \mathrm{l}$ of B. orientalis-infected blood by using TIANamp Genomic DNA Kit (Tiangen Biotech) by following the manufacturer's instructions and then stored at $-20{ }^{\circ} \mathrm{C}$ until further analysis.

\section{Cloning and sequencing of the truncated and full-length BoSBP3-like}

The BoSBP3-like gene was amplified from both gDNA and cDNA by PCR using the specific primers (SBP3$\mathrm{F} / \mathrm{R})$ listed in Table 1 . The specific primers were designed based on genome sequence of $B$. orientalis [17]. The thermal cycling parameters included the activation of Taq polymerase at $95{ }^{\circ} \mathrm{C}$ for $5 \mathrm{~min}, 33$ cycles of (denaturation at $94{ }^{\circ} \mathrm{C}$ for $30 \mathrm{~s}$, annealing at $55{ }^{\circ} \mathrm{C}$ for $30 \mathrm{~s}$, extension at $72{ }^{\circ} \mathrm{C}$ for $3 \mathrm{~min}$ ), and a final extension of $10 \mathrm{~min}$ at $72{ }^{\circ} \mathrm{C}$. The three truncated fragments of BoSBP3-like gene were amplified from gDNA using the same PCR procedure mentioned above with three different specific primers (SBP3-like-F1/R1, SBP3-like-F2/R2 and SBP3-like-F3/ R3) listed in Table 1.

The amplicons were electrophoresed using 0.8\% ethidium bromide-stained agarose gel and purified by EasyPure $^{\bullet}$ Quick Gel Extraction Kit (Invitrogen). The purified DNA fragments were ligated into pMD19-T vector (TaKaRa Biotechnology, Beijing, China). The BoSBP3-like sequence and the absence of introns were confirmed by isolating and sequencing the positive

Table 1 Primers used for the subcloning and sequencing of recombinant plasmids

\begin{tabular}{lll}
\hline Primer & Primer sequence (5'-3') & $\begin{array}{l}\text { Restriction } \\
\text { endonuclease }\end{array}$ \\
\hline SBP3-like-F & ATGAGGCGCTTCACCCTGCTAGCGTTG & \\
SBP3-like-R & TTACATGTCCATACCGACGCGGCATAG & \\
SBP3-like-F1 & CGGATCCATGAGGCGCTTCACCCTG & BamHI \\
SBP3-like-R1 & GCGTCGACAATCTGAGTGAAGTTGACG & Sall \\
SBP3-like-F2 & GGAATTCATGGCCCCACCTTCTGCC & EcoRl \\
SBP3-like-R2 & GCGTCGACTTACTGGAGGAAGAACTGG & Sall \\
SBP3-like-F3 & CGGATCCATGATCACTGCTTACGAC & EcoRl \\
SBP3-like-R3 & GCGTCGACTTACATGTCCATACCGACG & Sall \\
\hline
\end{tabular}

plasmids (Tianyi Huiyuan Biological Technology, Wuhan, China).

\section{Bioinformatics analysis}

The amino acid sequence of BoSBP3-like was translated using the ExPASY online tool (http://www.expasy.org/ translate/). Protein sequences were analyzed by predicting the transmembrane (TM) regions using TMHMM 2.0 (http://www.cbs.dtu.dk/services/TMHMM-2.0/) and predicting the putative glycosylphosphatidy-l-inositol (GPI) anchors using GPI Prediction Server 3.0 (http:// mendel.imp.ac.at/sat/gpi/gpi_server.html). BioEdit software was used to analyze the putative signal peptide (http://www.cds.dtu.dk/dervices/signalp/) and the potential subsequence motifs (http://mythis.isb-sib.ch/cgi-bin/ motif_scan) in BoSBP3-like by combining the results of multiple sequence alignment among $B$. orientalis, $B$. bovis and $B$. bigemina. The secondary structure and the 3D-structure of BoSBP3-like were subsequently predicted by DNAstar software and I-TASSER standalone package, respectively [18-20].

\section{Truncated expression and purification of the recombinant proteins}

The length of the BoSBP3-like gene is $3237 \mathrm{bp}$, which was too long for prokaryotic expression. The ORF of BoSBP3-like gene was truncated into three fragments: BoSBP3-like-1 (915 bp), BoSBP3-like-2 (1311 bp) and BoSBP3-like-3 (1011 bp), which were amplified and cloned into the expression vector $\mathrm{pET}-28 \mathrm{a}$. The recombinant plasmids (pET-28a-BoSBP3-like-1, pET-28a-BoSBP3like-2 and pET-28a-BoSBP3-like-3) were transformed separately into E. coli BL21 (DE3) strain. Small-scale culture was subjected to $1 \mathrm{mM}$ isopropyl- $\beta$-D-thiogalactopyranoside (IPTG) induction overnight at $28{ }^{\circ} \mathrm{C}$ to identify the capacity and the forms of expression by SDS-PAGE analysis. BoSBP3-like-1/-2/-3 were expressed as Hisfusion proteins, and purified using proteinPure Ni-NTA Resin (TransGen Biotech, Beijing, China) according to the manufacturer's instructions. Briefly, the transformed $E$. coli was washed three times with PBS ( $\mathrm{PH}=7.4)$, lysed by ultrasonication in binding buffer containing phenylmethylsulfonyl fluoride (PMSF), and then centrifuged at $15,000 \times g$ for $15 \mathrm{~min}$ at $4{ }^{\circ} \mathrm{C}$. Supernatants containing the rBoSBP3-like-1/-2/-3 were purified with the Ni-NTA Resin (TransGen Biotech).

\section{Preparation of polyclonal antibodies}

Briefly, $500 \mu \mathrm{g}$ of each purified rBoSBP3-like-1/-2/-3 in Freund's complete adjuvant (FCA, Sigma, San Francisco, CA, USA) was subcutaneously injected into three Japanese white rabbits (specific pathogen-free, 3 $\mathrm{kg}$ each), respectively. Booster injections with the same antigen in FCA (Sigma) were given on days 14, 
21 and 28. Fourteen days after the last immunization, serum from the immunized rabbits was collected and stored at $-20{ }^{\circ} \mathrm{C}$ for further use.

\section{Western blot}

For identifying the specific antigenicity of BoSBP3-like, rBoSBP3-like-1/-2/-3 were separated on 12\% SDSPAGE, blotted onto a nitrocellulose membrane (Merck, Kenny, NJ, USA) and blocked with 5\% (w/v) skimmed milk in phosphate buffered solution Tween-20 (PBST). The membranes were probed with the serum $(1: 100$ dilution) from the buffalo naturally infected with $B$. orientalis or with the serum from the negative control buffalo. Then, the membranes were incubated with BovIgG/HRP (1:2000) as secondary antibodies (Bioss, Beijing, China) and developed using the DAB method (ZSGB-BIO, Beijing, China) to identify whether the antibody response against $\mathrm{rBoSBP} 3$-like was elicited in $B$. orientalis-infected buffalo.

To determine the native BoSBP3-like in merozoites of $B$. orientalis, the anti-rBoSBP3-like-1/-2/-3 rabbit sera were prepared to react separately with the protein from the lysate of $B$. orientalis-infected buffalo erythrocytes and uninfected buffalo erythrocytes as follows: $0.5 \mathrm{mg}$ (per lane) was subjected to $12 \%$ SDS-PAGE, transferred to a nitrocellulose membrane, and probed with the rabbit serum against rBoSBP3-like-1, rBoSBP3-like-2 and rBoSBP3-like-3 (1:100 dilution), respectively. As controls, lysate of $B$. orientalis-infected buffalo erythrocytes and uninfected buffalo erythrocytes were reacted with the serum of naïve rabbits. The membranes were washed with Tris buffered saline Tween-20 (TBST) and then incubated with goat anti-rabbit IgG/HRP (1:1000) as secondary antibodies (Bioss).

\section{Indirect immunofluorescence assay}

For IFA, smears of $B$. orientalis-infected buffalo erythrocytes were prepared on slides, air-dried and then fixed in cold $50 \%$ acetone-50\% methanol solution for $30 \mathrm{~min}$ at $-20^{\circ} \mathrm{C}$. The rabbit polyclonal antirBoSBP3-like-1/-2/-3 (1:200 dilution) and the naïve rabbit serum (as controls) were used as the first antibody on the fixed smears separately and incubated at $37{ }^{\circ} \mathrm{C}$ for $1 \mathrm{~h}$. After washing four times with cold PBS, smears were incubated with goat anti-rabbit IgG conjugated to Alexa Fluor 594 (1:1000 dilution; Invitrogen) as the secondary antibody, followed by parasite nuclear staining using the DAPI stain (1:1000 dilution; Invitrogen). Another negative control consisted of the second antibody only without incubation with the primary antibody. Finally, the results were analyzed by fluorescence microscopy.

\section{Results}

\section{Identification of BoSBP3-like gene}

The BoSBP3-like gene was amplified from gDNA by using SBP3-like-F and SBP3-like-R primers (Table 1), and its full length was 3237 bp (Fig. 1). Sequence analysis indicated that BoSBP3-like encoded a polypeptide of 1079 aa, with a predicted size of $135 \mathrm{kDa}$. A $3237 \mathrm{bp}$ fragment was obtained by cloning and sequencing the entire sequence coding for amino acids (CDS) of BoSBP3-like from gDNA and cDNA (Fig. 1). These results indicated that BoSBP3like was intronless. The nucleotide sequence reported in this study has been submitted to GenBank with the accession number of KT947106.

\section{Bioinformatics analysis}

The complete nucleotide sequence of BoSBP3-like revealed 70 and $76 \%$ similarity with the SBP3 gene of $B$. bovis (T2Bo) and B. bigemina, respectively. The amino acid sequences of SBP3-like were aligned using BioEdit software, and the results showed 61.6 and $35.0 \%$ identity to $B$. bovis SBP3 and B. bigemina SBP3, respectively. Additionally, the SBP3 genes of these three Babesia species showed high similarity at the $C$ terminus.

The isoelectric point (IP) values of SBP3 of $B$. orientalis, B. bovis and B. bigemina were 6.495, 6.04 and 5.523, respectively. The high hydrophilicity and antigenic index suggested that all of them were good candidates for vaccine.

According to some bioinformatic criteria for identifying possible exported proteins, BoSBP3-like was predicted in

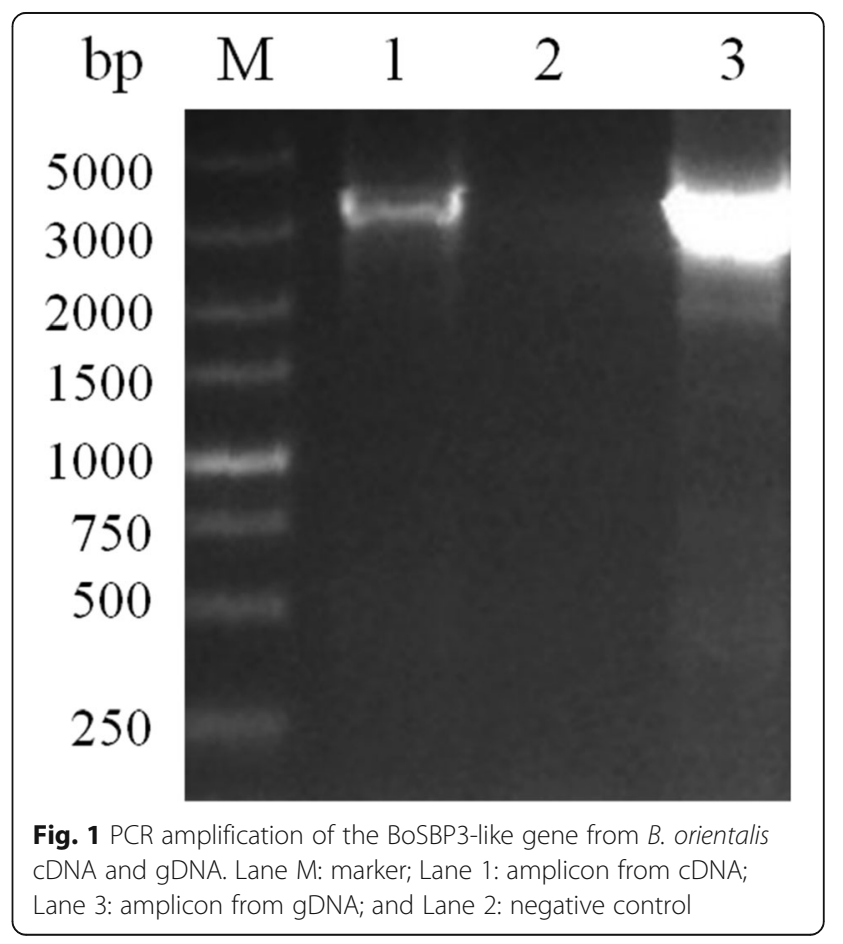


TM, GPI and signal peptide. As shown in the results of TMHMM 2.0 and GPI Prediction Server 3.0, BoSBP3-like had no TM regions or any GPI anchors, which was consistent with SBPs in B. bovis. Following the similarity with SBPs in B. bovis, BoSBP3-like was revealed to contain a signal peptide (1 to $16 \mathrm{aa}$ ), a CYS-rich region and three FAINT domains. According to the predicted information, the motif of BoSBP3-like is shown in Fig 2. A comparison of the predicted 3D-structure of $B$. orientalis and B. bovis (Fig. 2) was also made to find the conserved regions and the specific domains in the surface and some NAG drug sites.

\section{Truncated cloning and expression of BoSBP3-like}

According to the sequence analysis results, the BoSBP3like gene was truncated into three fragments: BoSBP3-like1 (915 bp), BoSBP3-like-2 (1311 bp) and BoSBP3-like-3 (1011 bp). The truncated fragments were expressed as His-fusion protein, and the sizes of rBoSBP3-like-1 (1305 aa), rBoSBP3-like-2 (306-742 aa) and rBoSBP3like-3 (743-1079 aa) were 43, 58 and $52 \mathrm{kDa}$, respectively. All rBoSBP3-like-1/-2/-3 were expressed in pellets. The rBoSBP3-like-1/-2/-3 were purified using Protein Pure Ni-NTA Resin (Fig. 3), then collected and dialyzed to immune rabbits.

\section{Identification of the recombinant and native BoSBP3-like} The specific antigenicity of BoSBP3-like was identified by western blot through the reaction of rBoSBP3-like$1 /-2 /-3$ with the serum collected from the $B$. orientalisinfected buffalo, with the serum of the normal buffalo used as a control. Three specific bands corresponding to rBoSBP3-like-1 (43 kDa), rBoSBP3-like-2 (58 kDa) and
rBoSBP3-like-3 (52 kDa) were detected in the serum from the $B$. orientalis-infected buffalo, but not in the serum of the control buffalo (Fig. $4 \mathrm{a}$ ).

The native BoSBP3-like was also determined by western blot through the reaction of the anti-rBoSBP3-like-1/-2/-3 immune serum from the rabbits with the $B$. orientalis-infected buffalo erythrocytes, and the control buffalo erythrocytes. A band about $135 \mathrm{kDa}$ was present in the $B$. orientalis lysate (Fig. $4 \mathrm{~b}$ ), which was consistent with the expected molecular weight of mature BoSBP3-like, but no band was observed in the negative controls. The other band may be attributed to the precursor of BoSBP3-like. The band of $135 \mathrm{kDa}$ was detected by western blot only in the presence of anti-rBoSBP3-like-1 immune serum, rather than anti-rBoSBP3-like-2/-3 immune serum, indicating that the immunogenicity of rBoSBP3-like-1 may be better than rBoSBP3-like-2 and rBoSBP3-like-3.

\section{Localization of BoSBP3-like}

The cellular localization of BoSBP3-like was determined using the polyclonal antibody generated from rabbits with the rBoSBP3-like-1/-2/-3. IFA was used to examine both the intracellular and extracellular parasite stages. However, the fluorescence of BoSBP3-like was only detected using rBoSBP3-like-1, rather than rBoSBP3-like$2 /-3$, which was consistent with the result of western blot in that only anti-rBoSBP3-like-1 immune serum from the rabbits reacted with the $B$. orientalis-infected buffalo erythrocytes.

In the extracellular parasite stage, fluorescence of BoSBP3-like was detected as puncta-like protein specks close to the nucleus of parasite. In the early intracellular parasite stage, BoSBP3-like was also detected as a

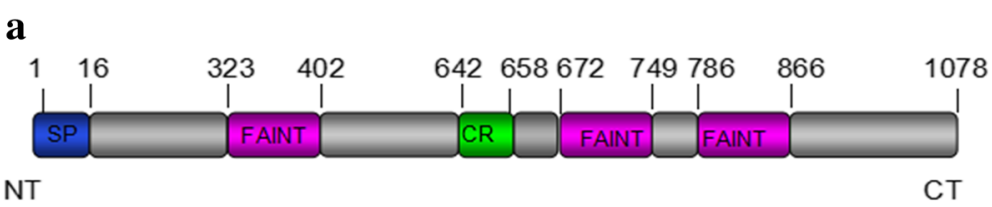

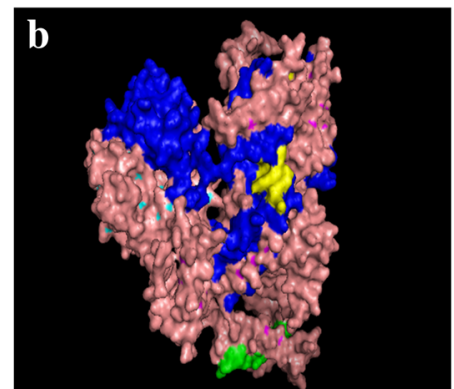

B. bovis

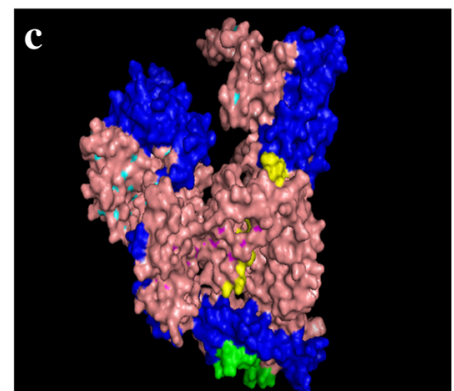

B. orientalis

Fig. 2 Schematic illustrations of predicted domains and crystal structures of SBP3. a Predicted domains of BoSBP3-like. A signal peptide (1-16 aa) at the N-terminus, three FAINT domains and a cysteine rich region. $\mathbf{b}$ Predicted crystal structure of SBP3 of B. bovis showing a signal peptide (yellow), three FAINT domains (blue) and one cysteine rich region (green). c The predicted crystal structure of BoSBP3-like 


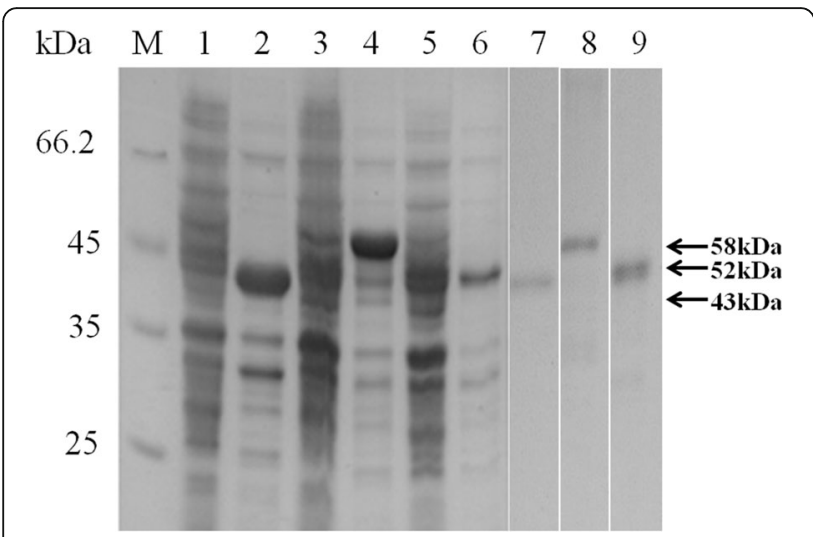

Fig. 3 SDS-PAGE of expressed and purified rBoSBP3-like-1/-2/-3. Lane M: molecular weight marker; Lane 1, Lane 3 and Lane 5: lysate of un-induced plasmids pET-28a-BoSBP3-like-1/-2/-3; Lane 2, Lane 4 and Lane 6: lysate of IPTG induced pET-28a-BoSBP3-like-1/-2/-3; Lane 7, Lane 8 and Lane 9: purified rBoSBP3-like-1/-2/-3. The corresponding bands are indicated by arrows

puncta-like protein speck close to the nucleus of parasite. Then, the fluorescence of puncta-like protein specks of BoSBP3-like was detected throughout the cytoplasm of iRBC at both single and paired parasite development stages, while no fluorescence was observed in the uninfected RBCs and the other two negative controls. Despite the confirmation of the secretion of BoSBP3-like to

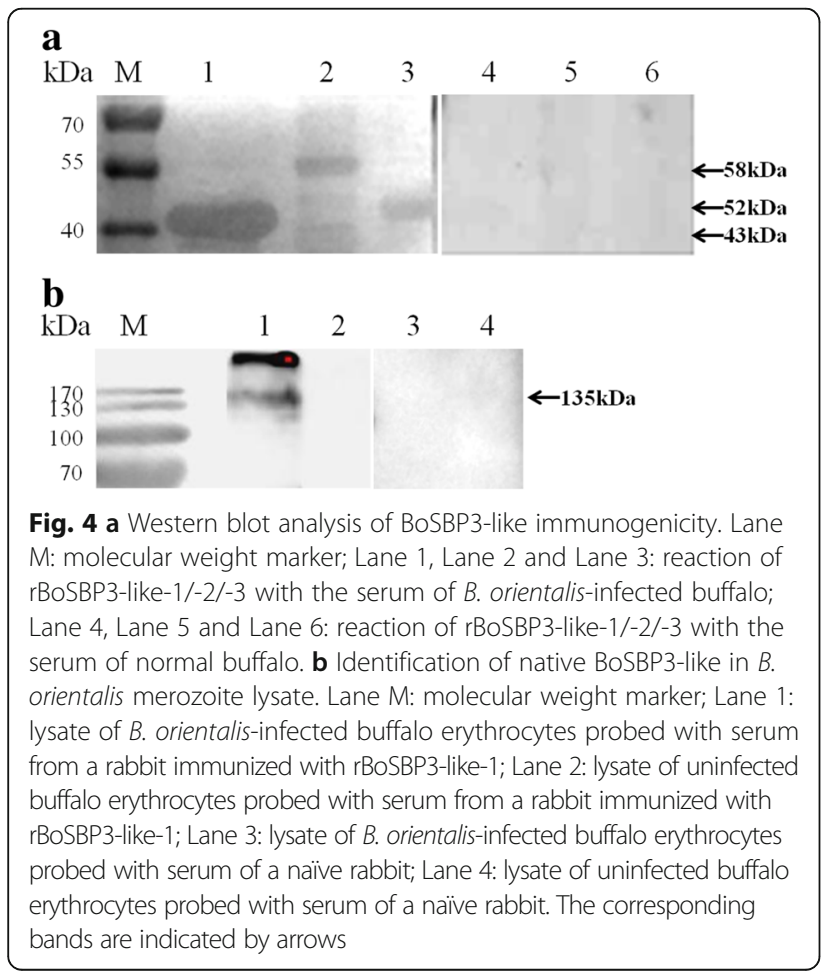

the cytoplasm of $\mathrm{iRBC}$, whether or not the secretion is on the cytoplasmic face of $\mathrm{iRBC}$ remains to be further elucidated (Fig. 5).

\section{Discussion}

This study has identified and characterized a novel 135 $\mathrm{kDa} B$. orientalis spherical body protein like, which shared a significant similarity to the SBP3 of B. bovis, and no critical amino acid identity to the other members of the SBP family (SBP1, SBP2 and SBP4). The significant similarity indicated that the identified gene was the BoSBP3-like gene. The BoSBP3-like was not only localized in the spherical body, but also in the cytoplasm of infected erythrocytes at both single and paired parasite development stages.

For elucidating the pathogenesis mechanism, there are two major processes for apicomplexan parasites: invasion and post-invasion. For the invasion stage, many efforts have been made to identify and characterize the groups of proteins released by apical complex, such as apical membrane antigen-1 (AMA-1), merozoite surface antigen (MSA), and Thrombospondin-related anonymous protein (TRAP) [21-23]. For the post-invasion stage however, little is known about the mechanism and related proteins involved in it. After invasion, iRBC will be altered in morphology and biochemistry. In morphology, iRBC will be more rigid, approximately three-fold higher than uninfected $\mathrm{RBC}$ in the mean value of the membrane shear elastic modulus [24], probably due to the presence of the non-deformable, abnormal intracellular parasite and modification of the RBC membrane skeleton proteins. On the other hand, the membrane of iRBC will be studded with ridge-like protrusion "ridges" which are described as knobs in Plasmodium falciparum. However in Babesia, ridges are found only present in B. bovis, but absent in B. bigemina, which may be related to pathogenicity [24]. In biochemistry, alterations in the $i R B C$ adhesive properties occur in the adhesion of healthy uninfected RBCs and the number of other cell types, especially vascular endothelial cells, leading to sequestration and reduction of clearance by spleen. The reason for altered adhesive properties may be related to modifications of iRBC membrane components. Due to lack of protein synthesis pathways of differentiated erythrocytes, all of the modifications of membrane components will be derived from parasites. In Plasmodium falciparum, Plasmodium falciparum erythrocyte membrane protein-1 (PfEMP-1) and rifin have been identified and characterized as present on the face of iRBC [25]. While substantial efforts have been done to identify proteins on the surface of $\mathrm{RBC}$, no proteins have been identified to be expressed on the surface of iRBC in Babesia. Currently, Babesia SBPs have been characterized to be expressed at post-invasion and 


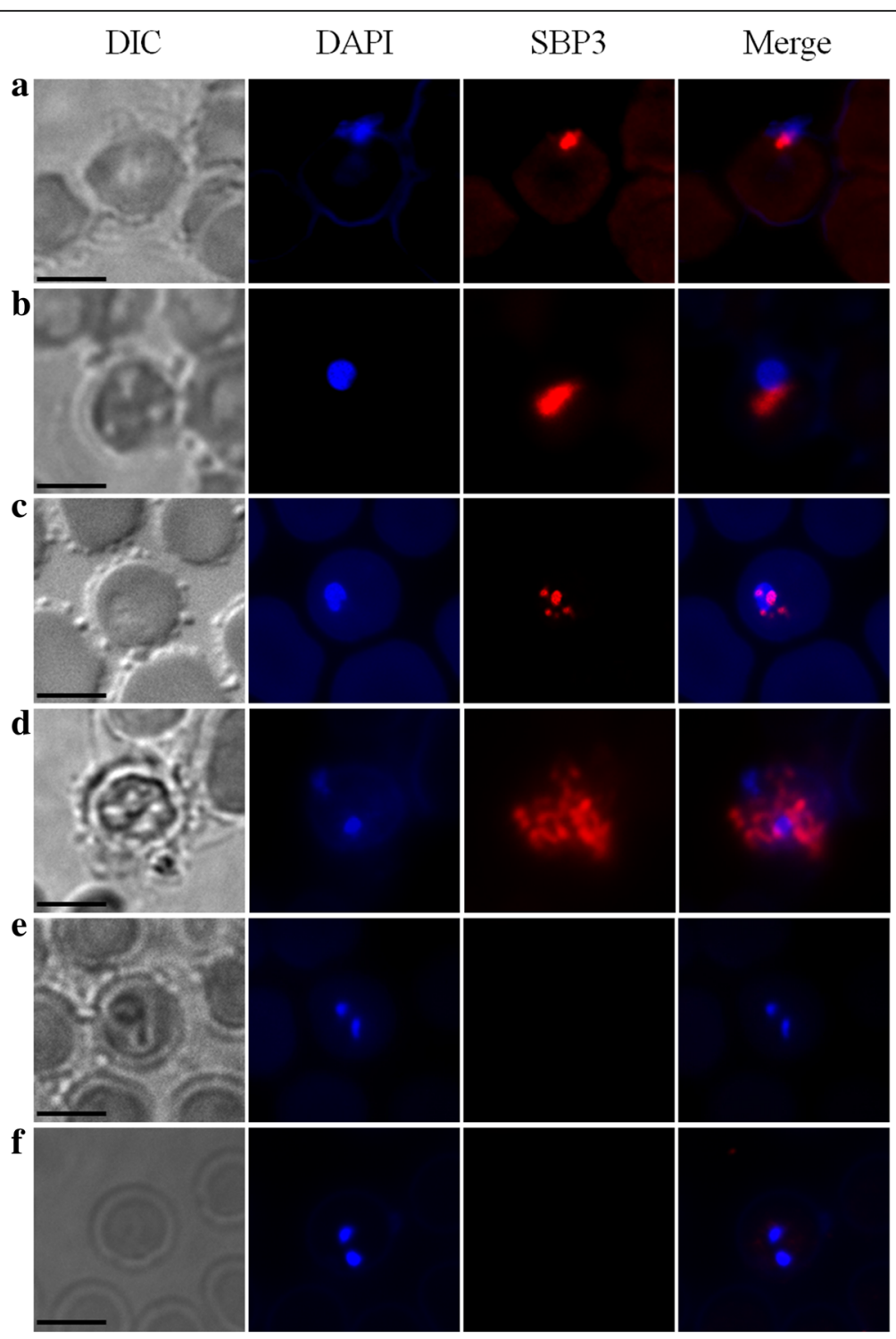

Fig. 5 Localization and distribution of BoSBP3-like. Immunofluorescence and electron microscopy analysis of BoSBP3-like in the smears. AntiBoSBP3-like serum (red) and nuclear staining of DAPI (blue). a Invasion stage. $\mathbf{b}$ The post-invasion stage of a single parasite. c At the post-invasion stage of a single parasite, BoSBP3-like was beginning to be secreted into the cytoplasm of iRBC. $\mathbf{d}$ At the post-invasion stage of paired parasites, BoSBP3-like was secreted into the cytoplasm of iRBC. e Negative control: the primary antibody was serum from a naïve rabbit. $\mathbf{f}$ Negative control: the second antibody only without incubation with the primary antibody. Scale-bars: a-f, $5 \mu \mathrm{m}$

secreted to the cytoplasmic face of iRBC. Whether SBPs are involved into the formation of ridges needs to be further investigated.

Spherical bodies are members of the apical complex and only exist in Babesia and Theileria, which share a similar function with dense granules of Plasmodium and other apicomplexan organisms $[2,6,7,9]$. Therefore, spherical body proteins may play a key role in host cell internalization. Notably, the parasites that contain dense granules all possess a parasitophorous vacuole (PV) during erythrocyte invasion, whereas PV will disintegrate after erythrocyte invasion in Babesia and Theileria, which contain spherical bodies but not dense granules [26]. These differences may indicate the possible relevance of disintegration of PV to spherical bodies. However, the association of disintegration of PV with spherical body proteins should be further investigated. In apicomplexan parasites, dense granule proteins participate in cell internalization and postinvasion process. For instance, ring-infected erythrocyte surface antigen (RESA) and ring membrane antigen (RIMA) are dense granule proteins of Plasmodium falciparum, which are released into the cytoplasm of iRBC and subsequently translocated to the cytoplasmic face of the iRBC [12]. Early immunoelectron microscopy 
and immunofluorescence studies showed that, in Babesia, only SBP1, SBP2 and SBP3 of B. bovis are secreted from spherical bodies, and subsequently released to the cytoplasmic face of the iRBC membrane at post-invasion $[1,6,7,27]$. Previous studies on the localization of SBP4 in $B$. bovis demonstrated that SBP4 was secreted in a stage-dependent manner, which only happened during the early stage of single merozoites, the ring-form stage and the early cell-division stage $[2,10]$. In this study, we identified the release of BoSBP3-like to the cytoplasm of iRBC for the first time. By indirect immunofluorescence assay, BoSBP3-like was detected not only close to the nucleus of the parasite but also throughout the cytoplasm of iRBC at both single and paired parasite development stages, demonstrating that BoSBP3-like was released into the cytoplasm of iRBC at all the parasite development stages. However, whether BoSBP3-like is released on the cytoplasm surface of iRBC has to be further studied. Meanwhile, a $135 \mathrm{kDa}$ band was observed in the lysate of $B$. orientalis by western blot, indicating the existence of a novel antigen SBP3-like in B. orientalis. The other band may be attributed to the precursor of BoSBP3-like, which also needs to be further investigated.

In the present study, the BoSBP3-like gene was identified from both gDNA and cDNA as an intronless gene, comparatively like in a $B$. bovis homologue, sharing a nucleotide identity of $70 \%$ in the amino acid coding region [6]. Due to its significant similarity to the SBP3 of $B$. bovis and B. bigemina, we defined the identified gene as the BoSBP3-like gene. Comparative analyses of SBP3 amino acid sequences from $B$. orientalis, $B$. bovis, and $B$. bigemina revealed a relative conservation at the $C$ terminus, indicating that SBP3 protein was conserved among Babesia. Additionally, BoSBP3-like was more close to B. bovis SBP3 in phylogenetic classification. Functional motif analysis revealed that SBP3 proteins were secreted into the host cytoplasm, and contained one to several FANIT domains. The FANIT domain was postulated to act as mediators of physiological changes associated with intracellular parasitism [10, 28]. Furthermore, SBP3 proteins in B. orientalis and B. bovis were revealed to contain a cysteine rich region, where structures were stabilized by disulfide bonds, which bypassed the more intensive selection needed to generate elaborate hydrogen-bonding interactions of complex $\alpha+\beta$ domain [29]. Previous studies have shown that cysteinerich regions are involved in intermolecular or molecular interactions by mediating binding contacts with several residues due to the surface localization and the charged nature in cysteine rich domains [30]. In the present study, we found the signal peptide of SBP3 protein present in both $B$. orientalis and $B$. bovis at the $\mathrm{N}$ terminus and displayed extracellularly, without TM regions or GPI anchors. This feature indicated that, as a secreted protein, BoSBP3-like processed a signal peptide that was necessary to guide the proteins into the secretory pathway and subsequently was exported to the infected erythrocyte. The absence of TM regions and GPI anchors meant that BoSBP3-like was not likely to be retained in the parasite. To understand whether there were any target sites in SBP proteins, we predicted and analyzed the 3D-structure of B. orientalis and B. bovis. Despite lack of available data on the full 3D-structure of the SBP3 protein, we constructed two predictive models using the I-TASSER software and $x$-ray diffraction in all existing protein databases. The combination of the predicted sequence characteristics, secondary structure motifs, partial domain localization in 3D-structure and similarity of SBP3 protein between $B$. orientalis and $B$. bovis led to the discovery of additional scientific information, which would facilitate further research on SBP3 protein functions. As illustrated, the signal peptide of the SBP3 protein in both $B$. orientalis and B. bovis was displayed at the $\mathrm{N}$-terminus, which enabled this protein to be secreted and localized specifically. The cysteinerich regions were also exposed at the same location, probably connected with their potential functions on molecular interactions. After protein 3D-structure prediction, NAG, a drug belonging to the anti-inflammatory category, was selected as the unique drug target based on the combined 3D-structure software prediction analysis of B. bovis and B. orientalis. Considering the involvement of BoSBP3-like in immune response, NAG component may be a potential effective drug for targeting BoSBP3-like.

In summary, we have identified and characterized a novel $135 \mathrm{kDa}$ spherical body protein-like of $B$. orientalis, with a significant similarity to SBP3 in B. bovis. It is localized in the spherical body of $B$. orientalis and discharged to the cytoplasm of iRBC at all parasite development stages. BoSBP3-like can serve as a candidate antigen for detection of $B$. orientalis. These findings may facilitate a comprehensive understanding of SBP3 and further related research.

\section{Conclusions}

The results of this article demonstrate that BoSBP3-like has a good immunogenicity and could act as a diagnostic antigen for $B$. orientalis. It was secreted into the cytoplasm of infected erythrocyte of buffalo at both single and paired shape parasite development stages, which indicates that BoSBP3-like might play a key role in postinvasion. This is probably the first report about identification and characterization of BoSBP3-like. However, the molecular mechanism of BoSBP3-like in the interaction with the host cell remains unclear and needs to be further investigated. 


\section{Abbreviations}

AMA-1: Apical membrane antigen-1; BoSBP3-like: The SBP3-like in Babesia orientalis; CDNA: Complementary DNA; CDS: Sequence coding for amino acids; gDNA: Genomic DNA; GPI: Glycosylphosphatidy-l-inositol; IFA: Indirect immunofluorescence assay; IP: Isoelectric point; IPTG: Isopropyl- $\beta$-Dthiogalactopyranoside; iRBC: Infected red blood cell; MSA: Merozoite surface antigen; NAG: N-acetyl glucosamine; ORF: Open reading frame; PBS: Phosphate buffer saline; PBST: Phosphate buffered solution Tween-20; PfEMP-1: Plasmodium falciparum erythrocyte membrane protein-1; PMSF: Phenylmethylsulfonyl fluoride; PV: Parasitophorous vacuole; rBoSBP3like-1-2/-3: Recombinant BoSBP3-like-1/-2/-3; RESA: Ring-infected erythrocyte surface antigen; RIMA: Ring membrane antigen; RT-PCR: Reverse transcribed polymerase chain reaction; SBPS: Spherical body proteins; SPF: Specific pathogen free; TBST: Tris buffered saline Tween-20; TEN: Tris/EDTA/NaCl; TM: Transmembrane; TRAP: Thrombospondin-related anonymous protein

\section{Acknowledgments}

Not applicable.

\section{Funding}

This study was supported by the National Basic Science Research Program (973 program) of China (Grant No. 2015CB150300), the National Key Research and Development Program of China (Grant No. 2017YFD0501201), the National Natural Science Foundation of China (Grant No. 31772729), and the Natural Science Foundation of Hubei Province (Grant No. 2017CFA020).

\section{Availability of data and materials}

All data are included as tables and figures within the article.

\section{Authors' contributions}

Collected the blood samples: JH, PH, ZN, ML, XZ and YZ. Performed the experiments: JG and JH. Participated in the data analysis: ML, LH, ZN, YS and LY. Helped with the diagnostic assays: PH and JH. Edited the manuscript: JL, $\mathrm{LH}$ and JZ. All authors read and approved the final manuscript.

\section{Ethics approval}

The experimental animals were housed and treated in accordance with the stipulated rules for the regulation of the administration of affair concerning experimental animals of the P. R. China. All experiments were performed under the approval of Laboratory Animals Research Centre of Hubei Province and the Ethics Committee of Huazhong Agricultural University (Permit number: HZAUCA-2016-007)

\section{Consent for publication}

Not applicable.

\section{Competing interests}

The authors declare that they have no competing interests.

\section{Publisher's Note}

Springer Nature remains neutral with regard to jurisdictional claims in published maps and institutional affiliations.

\footnotetext{
Author details

${ }^{1}$ State Key Laboratory of Agricultural Microbiology, College of Veterinary Medicine, Huazhong Agricultural University, Wuhan 430070, Hubei, China. ${ }^{2}$ Key Laboratory of Animal Epidemical Disease and Infectious Zoonoses, Ministry of Agriculture, Huazhong Agricultural University, Wuhan 430070, Hubei, China. ${ }^{3}$ Key Laboratory of Preventive Veterinary Medicine in Hubei Province, Wuhan 430070, Hubei, China. ${ }^{4}$ State Key Laboratory of Veterinary Etiological Biology, Key Laboratory of Veterinary Parasitology of Gansu Province, Lanzhou Veterinary Research Institute, Chinese Academy of Agricultural Science, Xujiaping 1, Lanzhou 730046, Gansu, China. ${ }^{5}$ Guangdong Laboratory Animals Monitoring Institute, Guangdong Key Laboratory of Laboratory Animals, Guangzhou 510663, Guangdong, China.
}

Received: 26 December 2017 Accepted: 13 March 2018

Published online: 27 March 2018

\section{References}

1. Gohil S, Herrmann S, Gunther S, Cooke BM. Bovine babesiosis in the 21st century: advances in biology and functional genomics. Int J Parasitol. 2013; 43:125-32.

2. Terkawi MA, Huyen NX, Wibowo PE, Seuseu FJ, Aboulaila M, Ueno A, et al. Spherical body protein 4 is a new serological antigen for global detection of Babesia bovis infection in cattle. Clin Vaccine Immunol. 2011;18:337-42.

3. He L, Liu Q, Quan M, Zhou DN, Zhou YQ, Zhao JL. Molecular cloning and phylogenetic analysis of Babesia orientalis heat shock protein 70 . Vet Parasitol. 2009;162:183-91.

4. Preiser $P$, Kaviratne $M$, Khan $S$, Bannister $L$, Jarra $W$. The apical organelles of malaria merozoites: host cell selection, invasion, host immunity and immune evasion. Microbes Infect. 2000;2:1461-77.

5. Bougdour A, Tardieux I, Hakimi MA. Toxoplasma exports dense granule proteins beyond the vacuole to the host cell nucleus and rewires the host genome expression. Cellular Microbiol. 2014;16:334-43.

6. Ruef BJ, Dowling SC, Conley PG, Perryman LE, Brown WC, Jasmer DP, et al. A unique Babesia bovis spherical body protein is conserved among geographic isolates and localizes to the infected erythrocyte membrane. Mol Biochem Parasitol. 2000;105:1-12.

7. Dowling SC, Perryman LE, Jasmer DPA. Babesia bovis 225-kilodalton spherical-body protein: localization to the cytoplasmic face of infected erythrocytes after merozoite invasion. Infect Immun. 1996:64:2618-26.

8. Jasmer DP, Reduker DW, Perryman LE, McGuire TC. A Babesia bovis 225kilodalton protein located on the cytoplasmic side of the erythrocyte membrane has sequence similarity with a region of glycogen phosphorylase. Mol Biochem Parasitol. 1992:52:263-9.

9. Hines SA, Palmer GH, Brown WC, McElwain TF, Suarez CE, Vidotto O, et al. Genetic and antigenic characterization of Babesia bovis merozoite spherical body protein Bb-1. Mol Biochem Parasitol. 1995;69:149-59.

10. Terkawi MA, Seuseu FJ, Eko-Wibowo P, Huyen NX, Minoda Y, AbouLaila M, et al. Secretion of a new spherical body protein of Babesia bovis into the cytoplasm of infected erythrocytes. Mol Biochem Parasitol. 2011;178:40-5.

11. Torii M, Adams JH, Miller LH, Aikawa M. Release of merozoite dense granules during enythrocyte invasion by Plasmodium knowlesi. Infect Immun. 1989;57: 3230-3.

12. Trager W, Rozario C, Shio H, Williams J, Perkins ME. Transfer of a dense granule protein of Plasmodium falciparum to the membrane of ring stages and isolation of dense granules. Infect Immun. 1992;60:4656-61.

13. Mercier C, Adjogble KD, Daubener W, Delauw MF. Dense granules: are they key organelles to help understand the parasitophorous vacuole of all Apicomplexa parasites? Int J Parasitol. 2005:35:829-49.

14. Diez-Silva M, Park Y, Huang S, Bow H, Mercereau-Puijalon O, Deplaine G, et al. Pf155/RESA protein influences the dynamic microcirculatory behavior of ring-stage Plasmodium falciparum infected red blood cells. Sci Rep. 2012;2:614.

15. He L, Feng HH, Zhang WJ, Zhang QL, Fang $R$, Wang $L X$, et al. Occurrence of Theileria and Babesia species in water buffalo (Bubalus babalis Linnaeus, 1758) in the Hubei Province, South China. Vet Parasitol. 2012;186:490-6.

16. Molloy JB, Bowles PM, Jeston PJ, Bruyeres AG, Bowden JM, Bock RE, et al. Development of an enzyme-linked immunosorbent assay for detection of antibodies to Babesia bigemina in cattle. Parasitol Res. 1998;84:651-6.

17. He L, Liu Q, Yao B, Zhou Y, Hu M, Fang R, et al. A historical overview of research on Babesia orientalis, a protozoan parasite infecting water buffalo. Front Microbiol. 2017:8:1323.

18. Burland TG. DNASTAR's Lasergene sequence analysis software. Meth Mol Biol. 2000:132:71.

19. Khan MK, He L, Zhang W, Wang Y, Tao Q, Song Q, et al. Identification of two novel HSP90 proteins in Babesia orientalis: molecular characterization, and computational analyses of their structure, function, antigenicity and inhibitor interaction. Parasit Vectors. 2014;7:293.

20. Zhang Y. I-TASSER server for protein 3D structure prediction. BMC Bioinformatics. 2008:9:40

21. Moitra P, Zheng H, Anantharaman V, Banerjee R, Takeda K, Kozakai Y, et al. Expression, purification, and biological characterization of Babesia microti apical membrane antigen 1. Infect Immun. 2015;83:3890-901. 
22. Rodriguez M, Alhassan A, Ord RL, Cursino-Santos JR, Singh M, Gray J, et al. Identification and characterization of the RouenBd1987 Babesia divergens Rhopty-associated protein 1. PLoS One. 2014;9:e107727.

23. Gaffar FR, Yatsuda AP, Franssen FF, de Vries E. A Babesia bovis merozoite protein with a domain architecture highly similar to the thrombospondinrelated anonymous protein (TRAP) present in Plasmodium sporozoites. Mol Biochem Parasitol. 2004;136:25-34.

24. Hutchings $C L$, Li A, Fernandez KM, Fletcher T, Jackson LA, Molloy JB, et al. New insights into the altered adhesive and mechanical properties of red blood cells parasitized by Babesia bovis. Mol Microbiol. 2007;65:1092-105.

25. Craig A, Scherf A. Molecules on the surface of the Plasmodium falciparum infected erythrocyte and their role in malaria pathogenesis and immune evasion. Mol Biochem Parasitol. 2001;115:129-43.

26. Rudzinska MA, Trager W, Lewengrub SJ, Gubert E. An electron microscopic study of Babesia microti invading erythrocytes. Cell Tissue Res. 1976;169: 323-34.

27. Gohil S, Kats LM, Seemann T, Fernandez KM, Siddiqui G, Cooke BM. Bioinformatic prediction of the exportome of Babesia bovis and identification of novel proteins in parasite-infected red blood cells. Int J Parasitol. 2013:43:409-16.

28. Anantharaman V, Iyer LM, Balaji $\mathrm{S}$, Aravind L. Adhesion molecules and other secreted host-interaction determinants in apicomplexa: insights from comparative genomics. Int Rev Cytol. 2007;262:1-74.

29. Cowman AF, Berry D, Baum J. The cellular and molecular basis for malaria parasite invasion of the human red blood cell. J Cell Biol. 2012;198:961-71.

30. Brayton KA, Lau AO, Herndon DR, Hannick L, Kappmeyer LS, Berens SJ, et al. Genome sequence of Babesia bovis and comparative analysis of apicomplexan hemoprotozoa. PLoS Pathog. 2007;3:1401-13.

\section{Submit your next manuscript to BioMed Central and we will help you at every step:}

- We accept pre-submission inquiries

- Our selector tool helps you to find the most relevant journal

- We provide round the clock customer support

- Convenient online submission

- Thorough peer review

- Inclusion in PubMed and all major indexing services

- Maximum visibility for your research

Submit your manuscript at www.biomedcentral.com/submit

C) Biomed Central 